\title{
POLYCENTER License System: Enabling Electronic License Distribution and Management
}

\author{
Timothy P. Collins \\ Digital Equipment Corporation \\ 153 Taylor St. , Littleton, MA 01460-1407 USA, \\ collins@nac.enet.dec.com
}

\begin{abstract}
License management is a neglected area of systems management. First-generation license systems have focused on preventing unauthorized software use. The POLYCENTER License System reaches out to provide an infrastructure for an electronic distribution chain, from software publishers and distributors to the end user. Novel security and customization features along with support for industry standard APIs make PLS convenient and safe to use.
\end{abstract}

Keywords

Software licenses, software asset management, LSAPI, public-key cryptography

\section{INTRODUCTION}

Software is usually licensed, not sold. The actual title or ownership of the software remains with its producer or publisher. The end user buys an agreement with the publisher to use the software and the media on which the software is delivered. This agreement, or software license, describes the conditions under which the user may run the publisher's software. A typical PC license agreement might state that the program may be installed on as many computer systems as is convenient for the end user so long as there is no possibility that the software could be used in two places at one time. This allows for the case where a user has both a home and a work machine. However, it forbids installing the software on a network for anyone to use. 
Software license constraints are seldom reasonable for the network administrator who needs the freedom to install software in locations which make the most sense for performance and storage management reasons. This can mean using a large and slow server for seldom used software packages, or redundant installations on several user workstations to facilitate legitimate (if temporary) transfers of licenses. End users frequently move their organizations into legal violation of software licenses. Most see little wrong with borrowing their neighbor's software if they need it to complete some task. The end result is widespread software piracy, occasional lawsuits (Didio, 1993), and the loss of billions of dollars of revenue worldwide for publishers.

First-generation license systems, such as Digital Equipment Corporation's License Management Facility (LMF) give useful assistance to the task of enforcing license agreements. Unless the user loads a unique license key for a program into a system, the program will not run. A variety of policies encoded into LMF help enforce those portions of the paper contract for which LMF can collect information. This can have dramatic effects on profitability. Recently Digital added software licensing to one of its $\mathrm{PC}$ products and increased revenues by tens of millions of dollars the first year.

Several years ago Digital embarked on a second generation license system project. The result of this effort is the POLYCENTER License System (henceforth PLS). PLS was developed to extend the reach of automated software licensing activities beyond simple enforcement of program use. Some of the key requirements were to:

- Support distributors: licenses are issued mostly by distributors, not publishers.

- Distribute software licenses across the network.

- Support centralized administration from a single management console.

- Support license policies which change faster than the software does.

- Recognize that sometimes it is just as important to allow unlicensed software to run as it is to stop it from running.

- Decryption-based security - where there is, in effect a secret password hidden in the system-was considered unwieldy and unsafe. Find something better.

The resulting PLS system met these requirements in the following way:

- Two kinds of licenses are supplied: license agreements and issuer agreements The latter is a license to create agreements, thus providing a distribution chain.

- Extraordinary levels of customization are possible.

- Public key cryptography, using the RSA algorithm, is used extensively to block forgery attempts. PLS is immune to reverse-engineering attacks.

- Usage logs provide a mechanism for system administrators to understand actual usage and overdraft events. Software purchases can be based on hard data.

- PLS conforms to the "License Service Application Programming Interface" (LSAPI, 1993) and will conform to proposed OMG and X/Open standards. 


\section{DISTRIBUTED LICENSE MANAGEMENT}

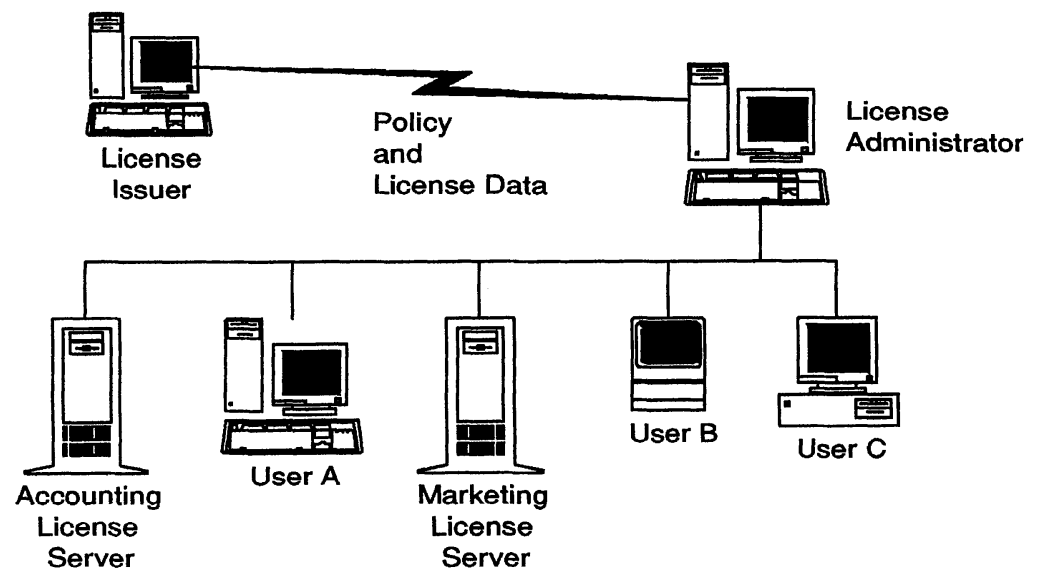

Figure 1 Network License Administration.

Figure 1 shows how a license is created and used. A license issuer creates licenses on their workstation using the PLS software. The licenses are held in the issuer's PLS database from which they may be extracted into one or more small ASCII files. These may then be copied to magnetic media or sent electronically (see Fenkel, 1993) to their destination. Along with supplying a GUI to create licenses interactively, PLS supplies an API through which the issuer's own order fulfillment system can create licenses automatically.

A license administrator may then load or "import" the licenses, can monitor usage of those licenses, and may set permissions on them. This includes entering user names or node names (or other features used for licensing) and "activating" the licenses. In the example in Figure 1 the licenses have been partitioned into those for the "accounting" and those for the "marketing" departments.

End user computers are configured with a list of license servers. These license servers will be searched for licenses when a licensed program is run. If the first server does not have the necessary resources then others on the list will be searched. This permits the license administrator to move licenses around the network or to reconfigure the network without adversely affecting the end users. Further, multiple servers help improve performance and mitigates any inconvenience resulting from unavailable servers.

Each license comes with a number of license units. A license unit is an arbitrary measure of value. One unit might represent one user. Alternatively, a license might come with 100 units but require that 10 units be used for a PC and 100 units for a mainframe. This is commonly termed a "capacity license." License units resemble currency printed by the license issuer. The value of the units from one issuer is 
unlikely to match the value of units from another. PLS ensures that units of unequal value are not mixed or combined.

Some portion of the license units supplied by a license are temporarily held reserved while a program is running and then released when the program exits. Should an application make a request, and all the license units are tied up in prior requests, then a failure reply will result. The application developer has the choice of determining how their program will respond. The program may exit back to the operating system, it may run but with reduced features, or it may ignore the result of the request. Consumptive licenses are also supported. For consumptive licenses the units are permanently deducted from the license rather than being released for use by others. This is good for trial licenses and for controlled pay-as-you-go metering styles.

\section{1 "License Service Application Programming Interface" ( LSAPI)}

The LSAPI defines a small number of calls which are to be added to an application program to be licensed (LSAPI, 1993). The benefit for the application programmer is that this job need be done only once. The specification was written by an assemblage of over 24 system and software vendors to serve as a standard license interface for application software. At the heart of the LSAPI are three calls:

Table 1 LSAPI Calls.

\begin{tabular}{|l|l|}
\hline LSRequest() & Ask permission to run. \\
\hline LSUpdate() & Program is still in use. Optionally, adjust license units used. \\
\hline LSRelease() & Program exiting, release held resources. \\
\hline
\end{tabular}

The LSRequest call is called by the application to receive initial permission to run. LSRequest supplies the publisher name, the product name, and the version number. These must uniquely identify a program.

The program may also request a particular license system to use, a suggested number of license units to use, a comment for whatever logging system might be present, and a challenge value. The LSAPI allows the client-side code to request either a specific license system or to try all of the license systems available using the reserved name "LS_ANY". This allows multiple license systems to coexist on the same network without requiring any intervention on the part of the end user.

The LSUpdate call is used to allow the application to check in with the license system to make sure that the original request is still valid. For example, if the license system were re-started, and the original request information lost, then too many users might be able to run. The update guards against this. It also represents an opportunity for the application to claim more license units as circumstances warrant. The final reason for an update request is to inform the license system that the application is still running and that the automatic time-out check should be re-started. 
The LSRelease call releases any resources held by PLS for others to use. It optionally supplies a number of license units to consume and a comment for whatever logging system which might be present. It also turns off the automatic time-out mechanism.

An optional challenge mechanism provides a way for the server and the application to believe that they are interacting with valid or authentic license system components. The challenge algorithm is based on the notion of shared secrets: a list of numbers both the client program and the license system both have but do not reveal to each other. The challenge mechanism can only be circumvented by a competent programmer who can examine the running code and determine how to extract the secrets either from the application or the server. These secrets could then be used for forge any number of perfectly acceptable licenses. For better security, PLS uses an additional technique-digital signatures using the RSA algorithm-described below in section 3.2 "Security Data".

\subsection{The Extended LSAPI}

The LSAPI does not supply a mechanism whereby the user's name, for example, can be collected and passed into the receiving license system. PLS supplies an optional field list mechanism to pass data in and out of a the normal LSAPI request, update or release calls. A field list is a list of paired name strings and value strings. A handful of routines are supplied which allow creating, adding to, modifying and destroying field lists. The LSAttach call tells the PLS software that a particular field list is to accompany a particular LSAPI request. Thus only the normal LSAPI interface is needed to communicate field lists with the license system.

An important use of the field list mechanism is to pass in the public key of the application's license data for verification purpose. This will be explored below in section 4. "Securing the Distribution Chain".

\subsection{Usage Enforcement Policies}

The first release of PLS on Windows NT comes with a small number of powerful enforcement policies. While more enforcement policies will be released for other platforms as well as for Windows NT over the next few months, these basic enforcement policies include:

- Concurrent Use. One unit held or deducted per request. Subscriber lists are provided for user and node names, but are ignored if not used.

- Node Lock. One unit held or deducted per request per node. A subscriber list is provided for node names. Change duration set by issuer.

- Personal Use. One unit held or deducted per request per user name. A subscriber list is provided for user names. Change duration set by issuer.

Other features included in the first release are support for the Dallas Semiconductor model DS1425 hardware security device, overdraft licenses, amendment licenses, 
capacity licenses, and embedded licenses. Any or all of these may be used with any of the three above enforcement policies, making the effective number of enforcement policies much greater.

An overdraft license is one which allows the creation of a 0 unit license to satisfy a request or an update. This allows the end user to always succeed in an attempt to use the software, and an overdraft event is added to the usage log. Allowing forgiving access to the license agreements is a distinct improvement over the normal pattern, which results in users quietly "borrowing" what they feel they need to do their jobs.

An amendment license agreement is a license agreement which seeks out another license agreement and replaces it Amendment license agreements let issuers correct mistakes to already deployed license agreements without having to issue new (and valuable) corrected license agreements.

Capacity license agreements compute the number of license units required for a request or an update by matching the requester's hardware in a list of possible hardware types. Thus, the a mainframe product can cost less if it runs on a PC.

\section{LICENSE DATA}

The PLS server database holds the objects which comprise both licenses and licenses to issue licenses. This section starts by describing how PLS objects may have their behavior customized (by adding rules and data fields) and secured against forgery (by receiving a digital signature.) Then the license agreement and issuer agreement objects are described.

\subsection{Policy Data}

First-generation license systems come equipped with a fixed repertoire of enforcement policies. They are typically coded in a third-generation language (C usually.) The license system code is typically split between the application program which makes the request and the license system which fulfills that request. The application may query the license system for information, interpret that information, and compute what to do next. The license system itself may be bound into the computer's operating system. For a description of a proposal to do this, see (Hauser, 1994).

The consequence of this is a very long lead time to make changes in the terms and conditions. Re-built software is usually only distributed to end users when there is a new release of that software. This bottleneck is felt keenly by distributors of electronic licenses, whether they are participants in the distribution chain or if they are trusted end users making their own electronic licenses. They simply don't have the source code and can never get it. If business, competitive, or legal circumstances require rapid changes in license policy they must wait for changes to be made by the application producer, the license system vendor, or both. This assumes they can persuade either party to change their code. 
PLS removes most of the policy computations for controlling use and issuing from all program binaries. Neither application programs nor the various management clients contain policy code. Further, PLS removes much of the policy computations from the PLS executables as well. Rather than encode key portions of the terms and conditions in C, a more "fourth-generation" approach was taken:

- An interpreted Pascal-like rule language allows distributors to author their own terms and conditions. These rules are invoked at key moments in processing usage requests and may control the success or failure of any operation. They may change field values to affect subsequent computations, and they may issue log events. Because these rules are interpreted and not compiled they work on any PLS system without modification.

- An extensible database which lets issuers define their own data fields on agreements, set them to initial values, and manipulate them through rules.

The data which comprises the rules and data field information is loosely termed policy data. Like all data in PLS it may be moved to a customer's computer via ASCII text files. The consequence of this is that new kinds of authorizations may be developed and installed in days or even hours. Contrast this with the worst case of using a license system bundled with an operating system released every 18 months.

\subsection{Security Data}

PLS uses the RSA public key cryptosystem algorithm for forgery protection and authentication. RSA was invented in 1977 by Ron Rivest, Adi Shamir, and Leonard Adleman (Rivest, Shamir, Adleman 1977). The RSA algorithm is used to compute a digital signature for PLS objects which protects them against forgery. See also (Tardo and Alagappan, 1990) and (ACM 1992).

The digital signature is based on a matched pair of keys (very, very large prime numbers.) One is termed the public key and accompanies the data to be signed where ever it goes. The other is termed the private key and is closely guarded. The RSA digital signature technique exploits a crucial property of the keys: they are 2way ciphers. This means that data encrypted with one key can only be decoded with the other key, and vice-versa. The algorithm is such that it is impossible (for all practical purposes) for an attacker to deduce one key from the other. Here's how a digital signature works.

First a digest of the data to be protected is computed. This is a large number (but much smaller than the message) which is a function of the value of the message. The digest has the property that a small change in the data causes a massive change in the number. Also, the message digest algorithm is designed so that it is impossible to create a reasonable-looking data stream which matches a given digest number. It is this digest value which is actually encrypted and decrypted. This reduces the amount of space consumed by the digital signature, as well as the amount of computation to encrypt and decrypt it.

Next the digest is encrypted with the private key and packaged with the data. We now have a digital signature. It gives us two valuable pieces of information about 
the object: who made the object (or changes to an object) and whether or not it was changed.

To see if someone changed the object we re-compute for ourselves the message digest. We then compare this new message digest with the old message digest. The old message digest is computed by decrypting the signature with the public key. If the old and new digests fail to match then the object has been changed. If they do match then only the holder of the private key could possibly have created the data and it is authentic.

\subsection{License Agreement}

License Agreement is the name given to the type of object that plays the role of an electronic license. A license agreement embodies all the constraints which determine whether or not a program may be run by a particular user. Once a user has actually been given permission to run under a license agreement then a license or grant is said to exist for a product. There can be no guarantee that a user has a license just because they have a license agreement. PLS must determine this.

All license agreements have at least these features:

- Start and end dates, plus an optional life span duration that begins after the license agreement is enabled for the first time.

- License units, plus a style field for allocative or consumptive accounting.

- A pair of license unit values to hold the number of units required for request or update calls respectively. A 0 value for either or both is permitted.

- An indication of the version(s) of the product.

- A selection weight value to be set by the license administrator. The license administrator might prefer to force the more selective licenses (such as the already assigned user license agreements, or individual product license agreements) to be used before the more general licenses (such as a concurrent use license agreement or a group license agreement.)

- A variety of subscriber lists for user or node names..

- Various title and comment fields allowing the license agreement to be selfdocumenting to a large degree.

To satisfy an LSRequest or LSUpdate call, PLS first locates all the license agreements which apply to that version. PLS then tests them to see if the user passes their usage constraints. These remaining candidate license agreements are then sorted in selection weight order. As many license agreements as are needed to get enough license units to satisfy the request are combined and their license units deducted. This information is held on the license data structure which keeps track of the user process which made the request, the product involved, and from which license agreements the units came. 


\subsection{Issuer Agreement}

Software developers seldom sell their software to end users directly. Most sell through a network of distributors. (See Figure 2.) For most PC software, far more than two thirds of the retail cost of software is a consequence of distribution chain markups. Finding ways to make software distribution channels both shorter and more efficient translates directly into cost savings for software buyers.

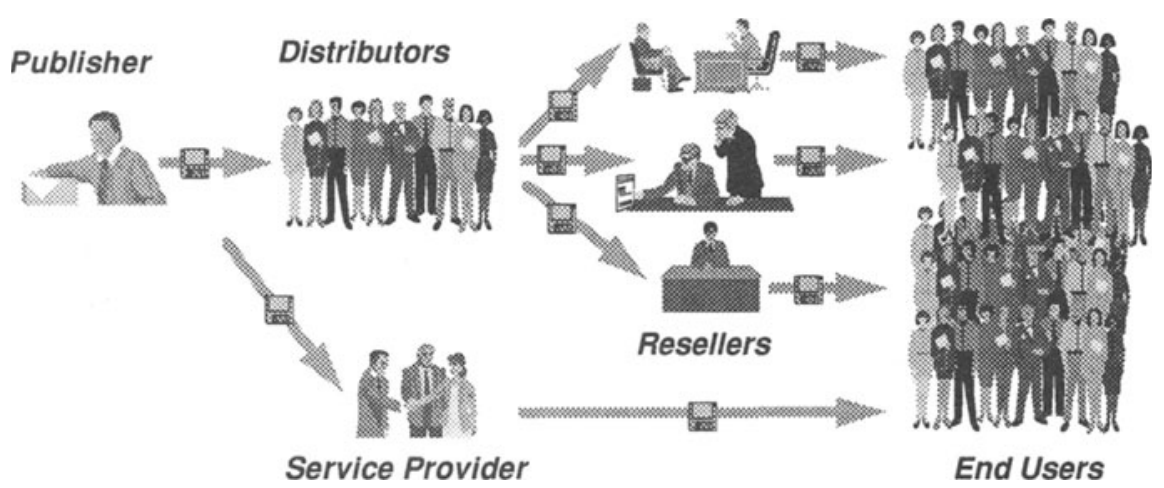

Figure 2 Software Distribution Chain.

The issuer agreement is a special sort of license agreement controlling the creation of either license agreements or subsequently issued issuer agreements. The issuer agreement specifies the kinds of usage constraints to follow, how many license units are available, and who may use those units. This other party may either make license agreements (and sell them to end users) or issuer agreements (and sell these to other issuers further down the distribution chain). PLS keeps a record of precisely which issuer agreement was the source of units for any subsequent license or issuer agreement. This constitutes an audit trail from the original producer, through any and all distributors or end-user issuers, to the final electronic license.

An interesting new channel for software distribution is the situation where the end user makes their own license agreements as they need them. The end user is trusted by their distributor to pay them fairly for what they take. Experiments by Digital with this model actually resulted in higher software revenues for the Digital and increased satisfaction on the part of the buyer. This reflects the sentiment that large corporate buyers want to be treated as partners and not adversaries, and will usually work faithfully to this end.

The first issuer agreement is termed the root issuer agreement and may specify an unlimited supply of license units. Issuer agreements created from other issuer agreements are termed sub-issued issuer agreements. 


\section{SECURING THE DISTRIBUTION CHAIN}

An object may have as an attribute the public key of another party from whom it is willing to accept as providing data to fulfill some role. Presuming no objects have been forged (which can be reliably proven) then the entire chain of objects which comprise the distribution chain can be authenticated using digital signatures.

- A producer can create a Product object providing the public key used to validate all license agreements and issuer agreements on the distribution chain.

- A producer can encode and protect "root" issuer agreements signed using the same key as the "product" object. They are, of course, entitled to issue agreements for their own products.

- An issuer agreement can specify the identity (public key) of another party on the next step of the distribution chain. This chain can be any length.

- An issuer agreement can specify the identity (public key) of another party entitled to make license agreements from their issuer agreement.

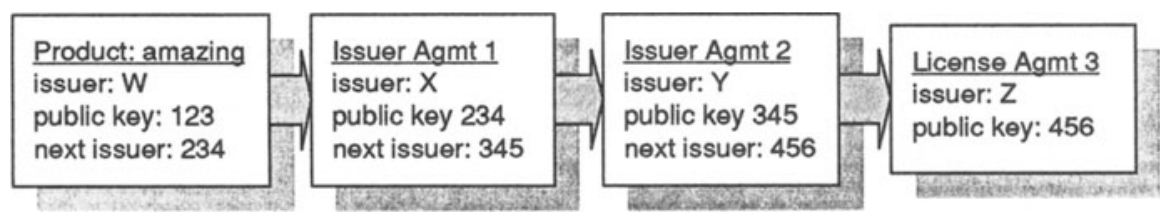

Figure 3 Securing the Distribution Chain.

We can now examine a sequence of digitally signed objects comprising the distribution chain for a single license agreement in Figure 3:

- Party W makes a Product indicating that the next issuer's public key for all "root" issuer agreements must be "234."

- Party X receives the Product and issues Issuer Agreement 1. Party $X$ is the only party possessing the private key portion which corresponds to the public key "234".

- Party Y receives Issuer Agreement 1 and issues Issuer Agreement 2. Party Y is the only party possessing the private key portion which corresponds to the public key of "345".

- Party $Z$ receives Issuer Agreement 2 and issues License Agreement 1. Party $Z$ is the only party possessing the private key portion which corresponds to the public key of "456."

Thus the entire distribution chain is protected by unbreakable RSA signatures: product to issuer agreement to optional sub-issuer agreement(s) to license agreements. The public keys prove that each object was created under authority of the one before. The series begins with the "product" entry in the license system.

How then is the public key for the "product" object to be trusted? What if some hostile issuer creates their own otherwise identical product object, and issues their own internally consistent chain of issuer and license agreements? The key is to find a trusted source for that public key from some source outside the license system. 
Privacy enhanced mail systems (such as Apple Computer's AOCE, or RSA Laboratory's TIPEM package) use an RSA certificate chain rooted at a TLCA ("top level certifying authority") to prove the authenticity of public keys. A RSA certificate is a signed document containing the names and public keys of parties whose public keys need to be verified. The TLCA provides a master RSA certificate containing the public keys needed to verify all the RSA certificates. This provides a single key value which, if unaltered, vouches for the integrity of all the other public keys. Such certificates are crucial for supporting public commerce using electronic means. For a cogent discussion of the value of digital signatures and the need for verifiable certificates nationwide see (Chokhani, 1994).

An alternate mechanism to verify public keys can eliminate the need for users to go to an outside party to have their public keys certified. The application program can pass the public key for the product object as part of the extended LSAPI LSRequest. The public key must match the public key on the product in the repository for the request to succeed. The rationale is that anyone wanting to substitute this key with one of their own could more easily patch around the LSRequest call instead. One way to look at a modification attack is to consider it equivalent to a viral invasion. Software licensing services do not protect against viruses which might damage license calls. Such services should be part of the underlying operating system instead.

\section{CONCLUSION}

Electronic licensing will, over the next few years, add more complexity to the task of managing resources in a network. Easy to use management tools will be necessary to ease the pain of introducing this emerging technology. At the same time licensing offers many benefits to the network administrator.

Software buyers can save money by developing a history of software use and from this knowing exactly how much software they need to buy. Perhaps every PC does not need a high-end word processor if a concurrent use license for only one-half of the PCs will get the job done. This should help cut down on the amount of "shelfware" sitting in people's bookcases. Buying quantities of licenses should garner discounts. This should be possible if the buyer is confident of the amount really needed. Finally, licensing should help reduce the expense of sudden, unexpected shifts of resources within a company. Rather than pay higher, retail prices in an emergency (such as a new hire, or a department started or shut down) the cheaper, bulk licenses can be rapidly deployed.

PLS raises the bar for all future license systems. Software publishers cannot be content with systems which do not provide a secure distribution channel into the marketplace. RSA digital signatures and PLS customization features supply the kind of enabling technology that electronic licensing needs. The advent of totally electronic distribution of software will cause major changes in the ways software is bought and sold. System managers need to start thinking now about what those changes will mean to them in the years to come. 


\section{REFERENCES}

ACM (1992). Special section on encryption standards and the proposed digital signature standard. Communications of the ACM, 35(7), pp. 36-54.

Chokhani, S. (1994) Toward a National Public Key Infrastructure. IEEE Communications Magazine, September, 1994,

Didio, L. (1993) Crackdown on Software Bootleggers Hits Home, LAN Times, 10(22).

Frenkel, G. (1993), Software Distribution: The Electronic Way, PC Magazine, September 28, pp. 11-16.

Hauser, R.C. (1994) Does Licensing Require New Access Control Techniques? Comunications of the ACM, 37(11), pp. 48-54.

LSAPI (1993), License Service Application Programming Interface, Version 1.1, December 14, 1993. Available by sending Internet mail tolsapi@microsoft.com, or by sending mail to Dave Berry, Microsoft Developer Relations, 1 Microsoft Way, $4 / 2$, Redmond WA 98052-6339, or from CompuServe, or from the author.

Rivest, R.L., Shamir, A., and Adleman, L. (1978) A method for obtaining digital signatures and public-key cryptosystems. Communications of the ACM, 21(2), 120-126.

Tardo, J. and Alagappan, K. (1990) Sphyinx: Global Authentication using Public Key Certificates. Digital Equipment Corporation.

\section{BIOGRAPHY}

Tim Collins received his BS in Zoology from the University of Massachusetts in Amherst in 1977. Over the last 17 years he worked as a scientific programmer, helped build CASE tools for structured analysis, tool integration, and configuration management, and was architect for PLS. Current interests include autonomous intelligent agents, OMG standards, object-oriented programming, and next-generation user interfaces. 